\title{
Análisis de la evolución diacrónica (1984-2014) de los fresnales de flor (Fraxinus ornus L.) valencianos mediante la interpretación de su interactividad geoecológica ("Método EFG-Meaza").
}

\section{Analysis of the diachronic evolution (1984-2014) of the flowering ash (Fraxinus ornus L.) forests of Valen- cia through the interpretation of their geoecologi- cal interactivity ("EFG-Meaza Method").}

David Cristel Gómez-Montblanch¹, Pedro José Lozano-Valencia²,

Emilio Laguna-Lumbreras ${ }^{3}$, Pedro Pablo Ferrer-Gallego 3, 4 , Guillermo Meaza-Rodríguez²

$\xi^{*}$

\section{Resumen}

En el presente trabajo se aborda el análisis de la influencia que ejercen los factores mesológicos -agrupados según la metodología propuesta ("Método EFG-Meaza")- para cada etapa de la sucesión vegetal en una comunidad de fresno de flor (Fraxinus ornus L.) de la Sierra de Malacara (Buñol, Valencia) estudiada por Laguna et al. (2015b) mediante su aplicación con carácter diacrónico (1984-2014). Se pretende comprobar si el grado de complejidad de las relaciones ecológicas estudiadas a la escala territorial propuesta -interactividad geoecoló-

\footnotetext{
1 Universidad de Sevilla

c/ Doña María de Padilla, s/n. 41004 Sevilla

Correspondencia: davidcristel@gmail.com

2 Universidad del País Vasco

c/Tomás y Valiente s/n. 01006 Vitoria-Gasteiz

Correspondencia: pedrojose.lozano@ehu.es, guillermo.meatza@gmail.com
}

3 Generalitat Valenciana. Centro para la Investigación y Experimentación Forestal

Avda. Comarques del País Valencià, 114. 46930 Valencia

Correspondencia: laguna_emi@gva.es

4 Generalitat Valenciana. VAERSA

Avda. Corts Valencianes, n²0.46015 Valencia.

Correspondencia: flora.cief@gva.es 
gica- guarda relación con la evolución de la comunidad concernida en cada etapa de sucesión, prestando especial atención a los cambios en su estructura y composición florística.

Palabras clave: interactividad geoecológica, "Método EFG-Meaza", fitoindicación, fitoacción, fenosistema, criptosistema, sucesión vegetal, fresnal.

\begin{abstract}
This paper sets out to analyse the influence that the mesologic factors -classified by the proposed method ("EFG-Meaza Method")- can apply at each stage of plant succession for one South European flowering ash community (Fraxinus ornus L.) studied by Laguna et al. (2015b) by means of its implementation on several dates over three decades with diachronic standpoint (1984-2014). The intention is, therefore to determine whether the degree of complexity of the ecological relations studied by the proposed landscape scale, called 'geo-ecological interactivity', relates to the evolution of the community studied at each stage of its plant succession, paying special attention to its structure and composition.
\end{abstract}

Key words: geo-ecological interactivity, "EFG-Meaza Method", phytoindication, phytoaction, phenosystem, cryptosystem, plant succession, ash wood.

\title{
Laburpena
}

Lan honetan zera aurkezten da, Laguna et al-ek (2015b) Malacara Mendizerran (Buñol, Valentzia) ikertutako loredun lizarraren (Fraxinus ornus L.) komunitate bateko landare jarraiera etapa bakoitzean faktore mesologikoek izandako eragina, proposatutako metodologiaren arabera taldekatuta (EFG-Meaza metodoa), garapen diakronikoaren bitartez (1984 eta 2014 artean). Egiaztatu nahi da ea proposatutako lurralde eskalan ikertutako erlazio ekologikoen konplexutasun maila -interaktibitate geoekologikoa- bat datorren jarraiera aldi bakoitzean inplikatutako komunitatearen eboluzioarekin, berebiziko arreta jarrita lore egituraketan eta osaketan gertaturiko aldaketetan.

Gako hitzak: Interaktibitate geoekologikoa, "EFG-Meaza metodoa", fitoadierazlea, fitoeragina, fenosistema, kriptosistema, landare jarraiera, lizardia.

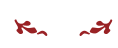

\section{Introducción}

La propuesta de Evaluación de la Funcionalidad Geoecológica comenzó a perfilarse en un trabajo de Meaza y Cadiñanos (2000) que valoraba el papel de la cubierta vegetal en la protección, equilibrio y estabilidad del medio en el que radica. Años más tarde, se publicó el trabajo "Fitoindicación/Fitoacción ambiental y territorial; ensayo de aplicación en la Reserva de la Biosfera de Urdaibai (País Vasco)" (Meaza \& Cuesta, 2010), en el que quedaron sentadas las bases del método "Fi/Fa" (acrónimo de Fitoindicación/Fitoacción) que, desde una perspectiva geoe- 
cológica del estudio de la vegetación, consideraba la interactividad entre las funciones delatora y controladora que aquella ejerce en relación a 5 parámetros mesológicos (geomorfológico, climático, hidrológico, edáfico y biótico), a los que se añadió la estrecha relación entre la vegetación y el ser humano (variable antrópica). En su virtud, y del mismo modo que se venía calificando como "fitoindicadoras" a las especies que asumen un rol pasivo, delator de las propiedades del medio, se proponía denominar - paralela y complementariamente- "fitoagentes" a las que ejercen un papel activo, controlador de las propiedades del medio. Era aquella una propuesta que, gracias a la aportación de nuevos investigadores, fue depurándose en sucesivas publicaciones: Gómez \& Meaza (2010); Gómez et al. (2010), Lozano et al. (2013), Gómez et al. (2014) y Gómez et al. (2015). Recientemente, se ha sustituído el acrónimo Fi/Fa por el más adecuado de EFG (Evaluación de la Funcionalidad Geoecológica).

En los trabajos antes indicados se aplicó la citada metodología a comunidades vegetales aisladas, o tomadas en uno o varios territorios de modo simultáneo, encajando en consecuencia en el estudio sincrónico de la vegetación. Otros trabajos, como los de Laguna et al. (2015a), donde se aportan inventarios de vegetación levantados secuencialmente en una misma zona en momentos temporales distintos, permiten abrir un nuevo campo de aplicación del método, relativo al estudio diacrónico de la vegetación. El inventariado diacrónico facilita una importante información sobre los procesos de la sucesión vegetal (Meaza, 2000) y, en el caso de las estimaciones del papel de la vegetación como fitoindicador y fitoagente, permiten conocer cómo evolucionan éstos en el tiempo. En el presente trabajo se ha aplicado el método EFG-Meaza al análisis de la evolución de los fresnales o fresnedas de flor valencianas en el período 1984-2014. Para la nomenclatura y autoría de los taxones se sigue el criterio de Flora Ibérica.

\section{Área de estudio}

La zona objeto del estudio la constituye el paraje de la Umbría del Fresnal (Buñol, Valencia), hemicuenca septentrional del Barranco del Fresnal, en el tramo occidental de la Sierra de Malacara o Montes de Buñol, en la provincia de Valencia (Fig. 1). Esta zona ha sido descrita en detalle por Laguna, y sus rasgos más relevantes se han sintetizado por Laguna et al. (2015a). La Umbría del Fresnal posee una superficie de 204 ha y altitudes comprendidas entre 605 y 964 m.s.n.m. El Barranco del Fresnal nace en el paraje del Collado Umán (812 m.s.n.m.) y discurre en sentido SE-NW (Fig. 2), por lo que las parcelas inventariadas se ubican con orientación a NNE (Laguna et al, 2015a). Se trata de una pendiente de terrenos básicos -derivados de materiales del Cretácico y Neógeno- orientada a N con ligero buzamiento NNW, y con vertido de aguas hacia el W, donde el Barranco del Fresnal se une a un curso mayor, la Rambla del Fresnal. Bioclimáticamente, conforme a los criterios de Rivas-Martínez et al. (2001), pertenece al piso Mesomediterráneo superior seco-subhúmedo, con tendencia al Supramediterráneo subhúmedo. En la clasificación corológica de Rivas-Martínez et al. (2001) se sitúa en el extremo nordoccidental del sector Setabense, dentro de la subprovincia Valenciana de la provincia Catalano-Provenzal-Balear. Se trata de un área de alta diversidad vegetal, con al menos 583 táxones de flora vascular hasta grado de subespecie (Laguna, 1997) y la vegetación dominante son pinares mixtos de Pinus halepensis, P. pinaster y P. nigra subsp. salzmannii, con sotobosque de frondosas -fundamentalmente Quercus ilex subsp. rotundifolia, Q. faginea subsp. faginea, Acer 


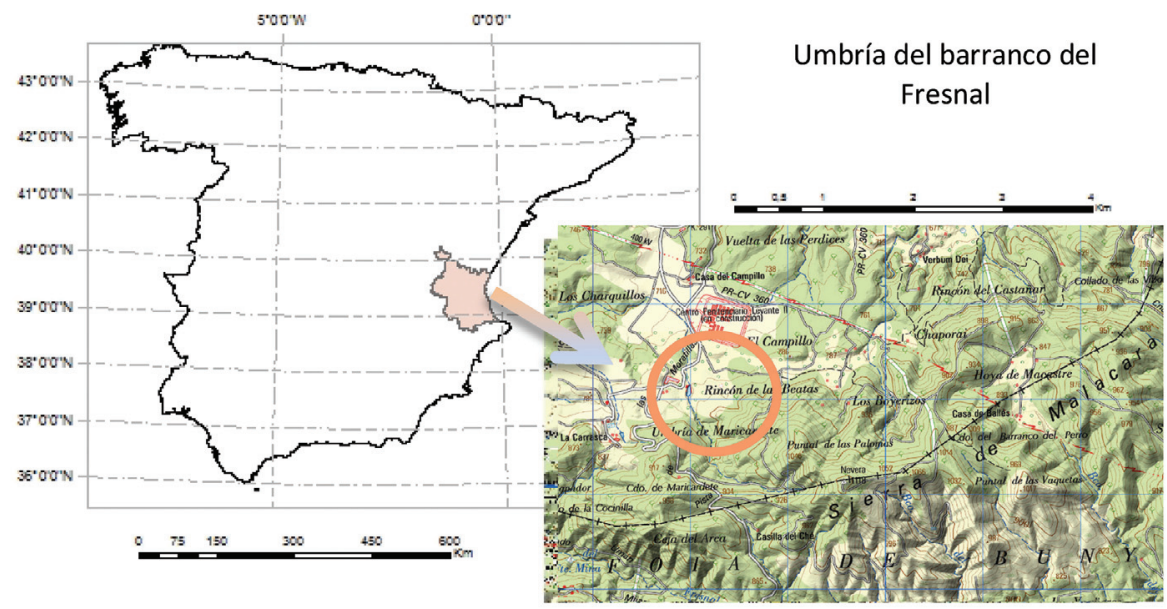

Fig. 1.- Localización general de la zona de estudio. Realizado por Itxaro Latasa.

Fig. 1.- Location of the study area. Created by Itxaro Latasa.

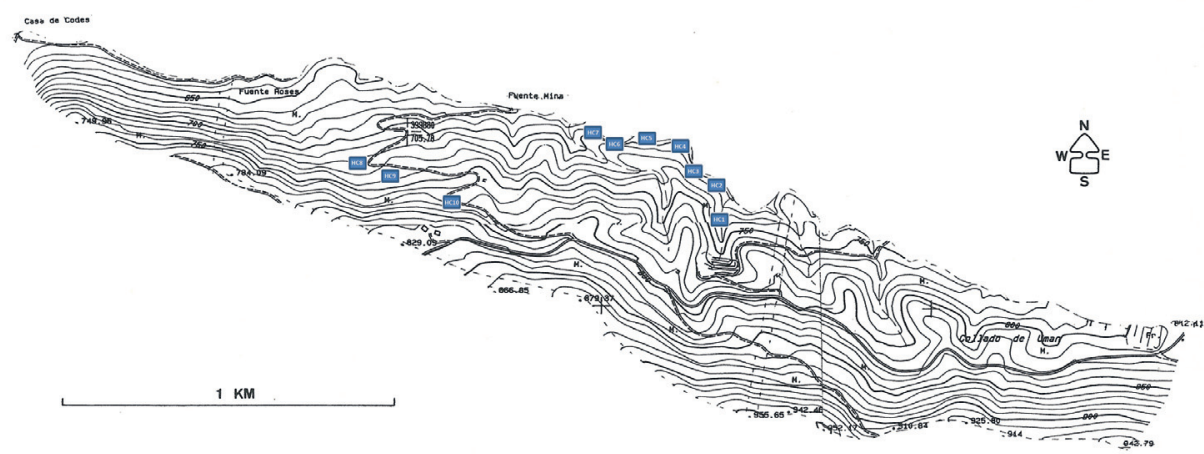

Fig. 2.- Topografía de la zona de estudio (isolíneas cada 10 m). En cuadrados resaltados, ubicación de las parcelas utilizadas para el seguimiento diacrónico de la vegetación.

Fig. 2.- Topography of the study area (10 m curves). Sampling plots highlighted in blue.

granatense y Fraxinus ornus, que llegan a ser dominantes en algunos rodales formando manchas de encinar, quejigar y bosquetes caducifolios. Dentro de estos últimos, la comunidad arbórea más relevante de la zona la constituyen los 'fresnales' o fresnedas de flor, dominadas por Fraxinus ornus; dentro de la Península Ibérica, esta formación vegetal aparece de modo natural casi exclusivamente en la Comunidad Valenciana (Rivas-Martínez et al., 2001., Laguna et al., 2015a). En 
el trabajo de Laguna et al. (2015a) se aportan detalles de su estructura y composición florística. La comunidad vegetal en la que encajan localmente las fresnedas de flor de la zona de estudio es el Hedero helicis-Cytisetum patentis Mateo 1982 subass. fraxinetosum orni Mateo 1982; RivasMartínez (2011) ha propuesto renombrar la asociación vegetal como Hedero helicis-Telinetum patentis Mateo 1982 nom. mut. propos. Rivas-Martínez 2011. La Umbría del Fresnal de Buñol alberga la localidad clásica de la subasociación ya indicada, que abarca los fresnales valencianos de óptimo mesomediterráneo.

\section{Metodología}

\section{Toma de datos de campo}

Como base para la aplicación del método EFG-Meaza, se ha trabajado sobre inventarios fitosociológicos del método Sigmatista o de Zürich-Montpellier (Braun-Blanquet, 1978), levantados sobre 10 parcelas de la comunidad Hedero helicis-Cytisetum patentis fraxinetosum orni, repetidos en los años 1984, 1994, 2004 y 2014, y realizados siempre por la misma persona. La ubicación y características sintéticas de las parcelas se aportan en el trabajo de Laguna et al. (2015a). Los sitios se corresponden además con los elegidos para el estudio sinfenológico de Laguna (1995), donde se incluyen descripciones más detalladas.

\section{Método de estimación e interactividad Fitoindicación/Fitoacción}

La metodología empleada en este trabajo ("Método EFG-Meaza") establece un protocolo evaluativo del interés mesológico de comunidades fitoindicadoras/fitoagentes en relación a 6 parámetros agrupados (climático, hídrico, geomorfológico, edáfico, biótico y antrópico), con objeto de conocer cómo las poblaciones que constituyen una comunidad vegetal interactúan con estos factores, tanto en su faceta delatora (fitoindicación) como en su rol agente sobre tales condiciones ambientales (fitoacción). Para ello, el proceso se inicia con la asignación de su rol ecológico en ambas facetas - delatora y agente- mediante el conocimiento de la autoecología de cada táxon. Una vez establecida dicha asignación, se inician los cálculos a partir de la ponderación del valor de AD para el rol fitoindicador-también podría emplearse valores de cobertura o número total de individuos, pues el método trabaja sobre poblaciones, aunque habría que ajustarla proporcionalmente respecto al conjunto de la fitocenosis-. En efecto, como luego se explica, la función fitoindicadora requiere de un tratamiento previo para discernir los bioindicadores más exigentes de los táxones más tolerantes a los factores ambientales concernidos por el método.

La propuesta metodológica que aquí se presenta constituye la versión más evolucionada y ambiciosa de cuantas se han aplicado desde hace casi seis años bajo la anterior denominación "Fi/Fa". Para ello, se ha reforzado el algoritmo y todas las funciones matemáticas adscritas al proceso, desarrollándose un apartado estadístico acorde a la exigencia científica correspondiente, haciendo posible su testado y evaluación en diferentes herramientas de modelización. La principal aportación del algoritmo (Fig. 3) reside en la equidad adoptada en las nuevas funciones propuestas para el estudio del carácter delator y condicionante de la vegetación. En tal sentido, se propone unificar la formulación de la siguiente manera: $\mathrm{Fi}_{\mathrm{f}}=\left({ }_{\Sigma} \mathrm{Fi}_{\mathrm{t}}{ }^{*} \mathrm{~K}\right) / \mathrm{NFi}$-en su caso 


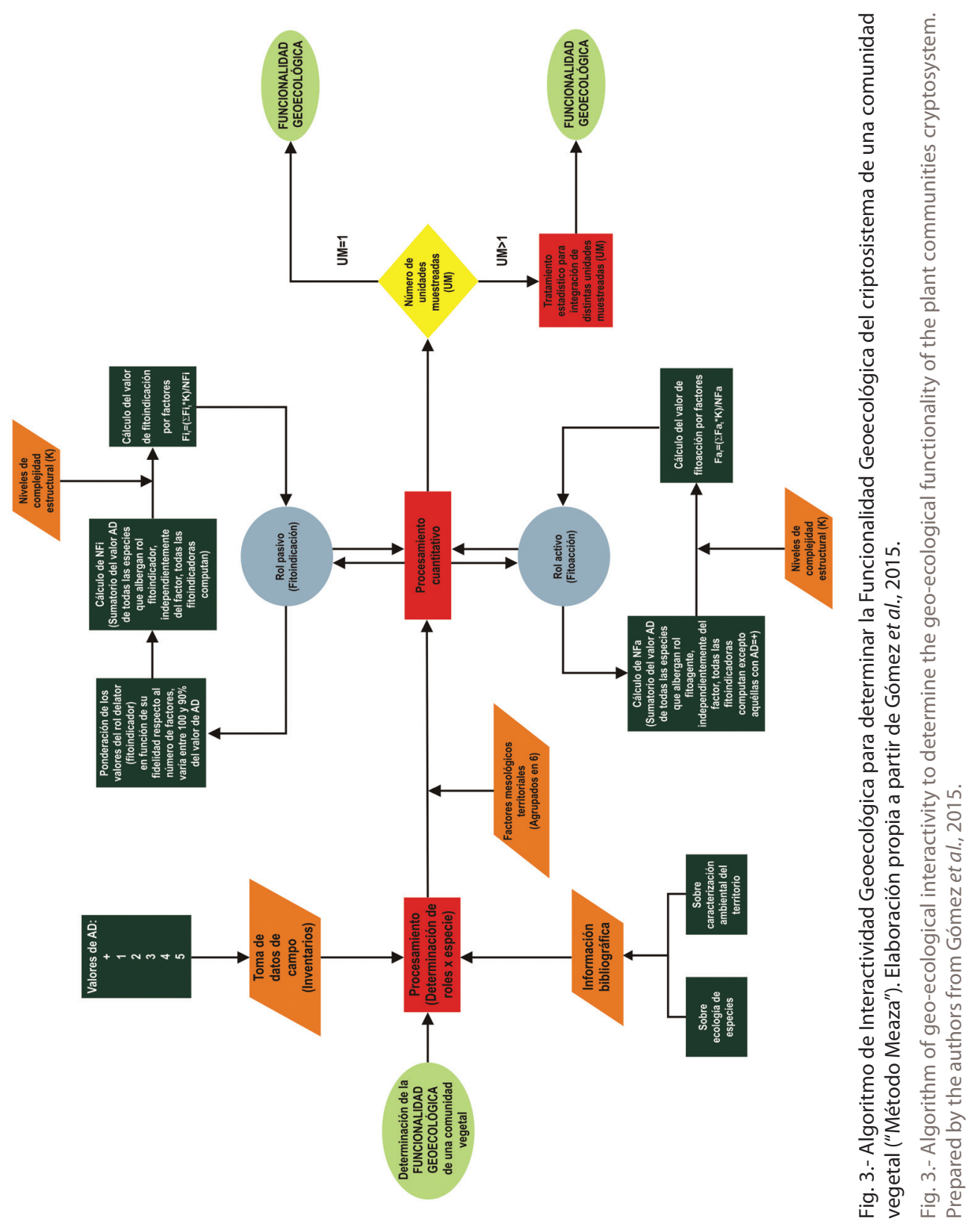


$\mathrm{Fa}_{\mathrm{f}}=\left(\sum_{\mathrm{F}} \mathrm{Fa}_{\mathrm{t}}{ }^{*} \mathrm{~K}\right) / \mathrm{NFa}$-. Como podrá observarse, en ambos casos se aprecia la inclusión de la constante de complejidad estructural (K) que varía en función de los parámetros descritos para 5 tipos de fenosistema vegetal posibles según Meaza \& Cuesta (2010) (Tabla 1). En la función propuesta se aprecia además que para el cálculo del valor de fitoindicación (Fi) -o en su caso fitoacción (Fa)- se necesita conocer el valor de fitoindicación -o fitoacción- calculado para cada factor para obtener el sumatorio con el que se establecerá el valor final incluyendo la constante $\mathrm{K}$-característica del fenosistema estudiado en ese determinado momento-y el valor obtenido de la suma de cobertura -en este caso obtenido por la aplicación del valor de AbundanciaDominancia (AD)- de los táxones que en el caso estudiado ejerzan un papel fitoindicador -o

\begin{tabular}{|c|l|}
\hline \multicolumn{2}{|c|}{ NIVELES DE COMPLEJIDAD ESTRUCTURAL } \\
\hline $\mathrm{K}$ & \multicolumn{1}{c|}{ TIPOS DE FENOSISTEMA } \\
\hline 1 & $\begin{array}{l}\text { Terrenos prácticamente desprovistos de vegetación; cultivos; vegetación temporal } \\
\text { o permanentemente sumergida; cultivos forestales intensivos mediante técnicas } \\
\text { silvícolas agresivas. Vegetación ruderal, arvense y de pisoteo; estadios prevascu- } \\
\text { lares y últimas etapas regresivas. Agrupaciones de xenófitas oportunistas y, en } \\
\text { general, vegetación desestabilizadora y depauperadora del sistema. }\end{array}$ \\
\hline 2 & $\begin{array}{l}\text { Vegetación herbácea o arbustiva rala; prados y herbazales sobrepastoreados; cul- } \\
\text { tivos y praderas abandonadas en primera fase de recuperación; vegetación perma- } \\
\text { nente herbácea primocolonizadora; cultivos forestales mediante técnicas no } \\
\text { agresivas. }\end{array}$ \\
\hline 3 & $\begin{array}{l}\text { Vegetación herbácea o arbustiva densa; pastizales o matorrales de uso ganadero } \\
\text { extensivo; disclímax, subclímax y piroclímax en fase de degradación menos acen- } \\
\text { tuada que la de la etapa anterior; vegetación permanente en fase avanzada; } \\
\text { vegetación arbórea rala con sostobosque ralo; plantaciones forestales antiguas. }\end{array}$ \\
\hline 5 & $\begin{array}{l}\text { Vegetación arbórea rala con sotobosque denso; vegetación arbórea densa con so- } \\
\text { tobosque ralo; disclímax, subclímax, plagioclímax, paraclímax, piroclímax y preclí- } \\
\text { max avanzados; vegetación permanente madura en medios dinámicos e } \\
\text { inestables; orlas y mantos; vegetación de ambientes ecotónicos; plantaciones } \\
\text { forestales históricas. }\end{array}$ \\
\hline $\begin{array}{l}\text { Vegetación arbórea densa con sotobosque denso; clímax, serclímax, quasiclímax, } \\
\text { peniclímax, plesioclímax y postclímax maduros; vegetación permanente madura } \\
\text { en medios muy dinámicos e inestables; vegetación de ambientes pluriecotónicos. } \\
\text { En general, ambientes vegetales de especial relevancia en la bioestabilización y } \\
\text { optimización de las condiciones ambientales }\end{array}$ \\
\hline
\end{tabular}

Tabla 1.- Niveles de complejidad estructural (K) de los tipos de fenosistema vegetal. Meaza \& Cuesta (2010).

Table 1.- Levels of structural complexity (K) for the types of plant phenosystems. Meaza \& Cuesta (2010). 
en su caso fitoagente- para cualquiera de los factores mesológicos agrupados, siendo éste, valor único divisor para el conjunto de factores evaluados en relación el carácter delator -o en su caso, condicionante-.

El valor de ponderación sobre el que se fragua la presente propuesta ha sido revisado para el rol fitoindicador, de manera que hubiese cierta penalización para los táxones menos restrictivos en su función delatora, y a su vez cierta compensación para los buenos fitoindicadores, minimizando además la lógica pérdida de peso específico respecto a la función fitoagente, en la que no se emplea tal ponderación puesto que se asume que la fitoacción se asocia al tamaño de la población de aquellos táxones considerados como tal.

Para ponderar la cuestión delatora en función de su fidelidad respecto al número de factores y conservar el peso de esta función en el conjunto del algoritmo que determina la funcionalidad geoecológica de las comunidades estudiadas, se ha establecido un umbral de ponderación que oscila entre el $90 \%$ y el 100\% del valor de AD de cada población inventariada (Tabla 2). De esta manera, se penaliza a los peores fitoindicadores, pero solo hasta un $90 \%$ del valor que aporta su población (AD) a la fitocenosis y minimizando así su pérdida de peso dentro del algoritmo.

\begin{tabular}{|c|c|c|c|c|c|c|c|c|}
\hline & \multicolumn{6}{|c|}{ NÚMERO DE VALORES } & \multirow{2}{*}{ POND } \\
\hline & & + & 1 & 2 & 3 & 4 & 5 & \\
\hline \multirow{6}{*}{ 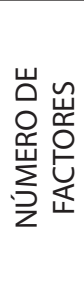 } & 1 & 0,1 & 1 & 2 & 3 & 4 & 5 & $100 \%$ \\
\hline & 2 & 0,098 & 0,98 & 1,96 & 2,94 & 3,92 & 4,90 & $98 \%$ \\
\hline & 3 & 0,096 & 0,96 & 1,92 & 2,88 & 3,84 & 4,80 & $96 \%$ \\
\hline & 4 & 0,094 & 0,94 & 1,88 & 2,82 & 3,76 & 4,70 & $94 \%$ \\
\hline & 5 & 0,092 & 0,92 & 1,84 & 2,76 & 3,68 & 4,60 & $92 \%$ \\
\hline & 6 & 0,090 & 0,90 & 1,80 & 2,70 & 3,60 & 4,50 & $90 \%$ \\
\hline
\end{tabular}

Tabla 2.- Ponderación de valores del interés delator (Fitoindicación). Elaboración propia.

Table 2.- Weighing coefficients for phyto-indication values. Prepared by the authors.

Una vez determinados los valores de fitoindicación y fitoacción para cada táxon y cada factor, éstos quedan ubicados en 12 columnas - 6 por cada factor respecto a fitoindicación y otras 6 respecto a fitoacción- y se procede entonces a sumar los valores establecidos por columnas para aplicar luego la previamente citada formulación $\left(\mathrm{Fi}=\left({ }_{\Sigma} \mathrm{Fi}_{\mathrm{t}}{ }^{*} \mathrm{~K}\right) / \mathrm{NFi}\right.$ o $\left.\mathrm{Fa} \mathrm{f}_{\mathrm{f}}=\left({ }_{\Sigma} \mathrm{Fa}_{\mathrm{t}}{ }^{*} \mathrm{~K}\right) / \mathrm{NFa}\right)$.

La versión actual del método incluye la utilización de ciertos estadísticos calculados a partir de los valores obtenidos para la fitoindicación y fitoacción respecto a cada uno de los factores mesológicos agrupados en las 6 categorías propuestas. En efecto, los estadísticos empleados fueron testados en un trabajo previo (Gómez et al., 2015) con datos de comunidades riparias de la cuenca del río Guadaíra en la Provincia de Sevilla. Estos estadísticos (Tabla 3) son empleados para obtener el "Rango de Interactividad" -que muestra la oscilación entre la mínima y máxima funcionalidad gecológica respecto a un determinado factor a partir del comporta- 


\begin{tabular}{|c|c|c|}
\hline ESTADÍSTICO & FORMULACIÓN & EXPLICACIÓN \\
\hline $\begin{array}{l}\text { Valor estimado de } \\
\text { Fitoindicación para } \\
\text { cada factor -en su } \\
\text { caso Fitoacción- }\end{array}$ & $\begin{array}{l}\overline{\mathrm{X}} \mathrm{Fi}_{f}=\left(\sum \mathrm{Fi}_{\mathrm{f}}^{*} \mathrm{~K}\right) / \mathrm{No} \\
\overline{\mathrm{X} F a}=\left(\Sigma \mathrm{Fa}_{f}^{*} \mathrm{~K}\right) / \mathrm{N} ; \\
\text { Donde } \mathrm{N} \text { es el número de } \\
\text { unidades inventariadas }\end{array}$ & $\begin{array}{l}\text { Se trata del valor medio de fitoindi- } \\
\text { cación -o en su caso fitoacción- } \\
\text { para cada factor en función del } \\
\text { número de inventarios realizados } \\
\text { para la caracterización de un tipo } \\
\text { de comunidad vegetal }\end{array}$ \\
\hline $\begin{array}{l}\text { Valor sobreestimado } \\
\text { de Fitoindicación para } \\
\text { cada factor -en su } \\
\text { caso Fitoacción- }\end{array}$ & 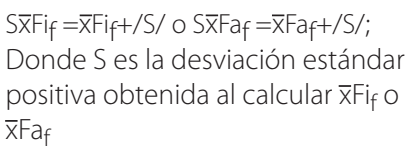 & $\begin{array}{l}\text { Es el valor resultante de sumar el } \\
\text { valor estimado (promedio) con su } \\
\text { desviación estándar de signo posi- } \\
\text { tivo para cada factor }\end{array}$ \\
\hline Amplitud estimada & $A_{e}=\left\{\left[\bar{x} F i_{f}-(K / 2)\right]+\left[\bar{x} F a_{f}-(K / 2)\right]\right\}$ & \multirow{2}{*}{$\begin{array}{l}\text { Se trata de un estadístico que mues- } \\
\text { tra la intensidad de la interactividad } \\
\text { entre factores en función de los roles } \\
\text { adquiridos por la comunidad vegetal } \\
\text { estudiada. Es necesario para el } \\
\text { cálculo del siguiente (Gradiente de } \\
\text { interactividad), considerando tan } \\
\text { solo a aquellos factores cuyo valor } \\
\text { sea superior a cero }\end{array}$} \\
\hline $\begin{array}{l}\text { Amplitud } \\
\text { sobreestimada }\end{array}$ & $A_{s}=\left\{\left[S \bar{x} F i_{f}-(K / 2)\right]+\left[S \bar{x} F a_{f}-(K / 2)\right]\right\}$ & \\
\hline $\begin{array}{l}\text { Gradiente de } \\
\text { interactividad }\end{array}$ & $\mathrm{Gi}_{e}=\mathrm{A}_{e} / \mathrm{K} \circ \mathrm{Gi}_{\mathrm{s}}=\mathrm{A}_{s} / \mathrm{K}$ & $\begin{array}{l}\text { El Gradiente de interactividad es un } \\
\text { valor normalizado que permite } \\
\text { comparar diferentes comunidades } \\
\text { vegetales independientemente de } \\
\text { su nivel de complejidad estructural } \\
\text { expresado por la constante K }\end{array}$ \\
\hline $\begin{array}{l}\text { Rango de } \\
\text { interactividad }\end{array}$ & $\mathrm{Ri}=\mathrm{Gi}_{\mathrm{e}}<\mathrm{Gi}_{\mathrm{s}}$ & $\begin{array}{l}\text { Permite apreciar la tendencia de in- } \\
\text { teractividad que podría manifes- } \\
\text { tarse en un futuro a tenor de lo que } \\
\text { indiquen las unidades muestreadas } \\
\text { en función del comportamiento de } \\
\text { sus medias respecto a los valores } \\
\text { díscolos -resuelto con la aportación } \\
\text { de la desviación estándar de signo } \\
\text { positivo-. Además permite com- } \\
\text { parar comunidades vegetales con } \\
\text { diferente complejidad estructural, al } \\
\text { tratarse de valores normalizados }\end{array}$ \\
\hline
\end{tabular}

Tabla 3.- Estadísticos empleados en la determinación de la funcionalidad geoecológica de la comunidad estudiada. Elaboración propia.

Table 3.-Statistical compilation used to analyze the geo-ecological functionality of the studied community. Prepared by the authors. 
miento delator y agente de la fitocenosis- con objeto de evaluar la intensidad de la interactividad geoecológica respecto a los factores más influyentes en la funcionalidad geoecológica de su criptosistema (González, 1982), permitiendo además su comparación con otras comunidades al tratarse de valores normalizados que oscilan en función de los valores de gradiente de interactividad obtenidos para cada valor estimado y sobreestimado -el valor sobreestimado resulta del cómputo entre el valor medio del conjunto de parcelas inventariadas en cada año y su desviación estándar de sentido positivo para cada rol y factor-.

\section{Tratamiento estadístico}

Con independencia de los cálculos de parámetros antes indicados, que se orientan especialmente a la representación gráfica de los resultados, se han comparado los valores de Fitoindicación y Fitoacción de los 6 variables o factores mesológicos (Climático, Hídrico, Geomorfológico, Edáfico, Biótico y Antrópico) con dos tipos de tratamientos:

- Sin combinar los valores de fitoindicación y fitoacción para una misma variable, se ha comparado la media de pares de muestreos sucesivos, mediante uso del estadístico $t$ de Student para distribuciones de datos apareados. Se analizaron los datos de las 3 transiciones decenales -de 1984 a 1994, de 1994 a 2004 y de 2004 a 2014- para las 12 variables -6 de fitoindicación y 6 de fitoacción-, para $n=10$ inventarios. Cada prueba posee 9 g.d.l. con un valor crítico de $t=2,2362$ para $p=0,05$.

- Combinando los valores de fitoindicación (Fi) y fitoacción (Fa). El parámetro analizado ha sido Fa - Fi para un mismo factor mesológico - p. ej., restando al valor de la Fitoacción climática de cada inventario el que se haya obtenido para la Fitoindicación climática-, realizándose:

a) La comparación de datos apareados para las transiciones entre muestreos, por el mismo método antes indicado, con uso del estadistico t de Student, aplicado a las 6 variables Fi - Fa (Climática, Hídrica, etc.).

b) La comparación completa de todas las muestras de un mismo factor mediante ANOVA simple, obteniendo tanto los valores para todo el recorrido temporal, como los de cada par de muestras -años- a través del estadístico Q de Tukey. Los ANOVA poseen 39 g.d.l. (3 entre grupos y 39 dentro de los grupos).

Para este último análisis se han realizado además las pruebas de Levene de homogeneidad de las varianzas y el test $F$ de Welch para la hipótesis de disimilaridad de al menos una de las varianzas.

Los fundamentos estadísticos de los tests y análisis realizados están detallados en obras generales (v. Sokal \& Rohlf, 1994), y los análisis se han realizado usando los paquete estadísticos PAST v. 3.10 (Hammer, Harper \& Ryan 2005; Hammer, 2014) y XLSTAT v. 8.0.

\section{Resultados}

Los resultados se presentan en una serie de 10 inventarios agrupados secuencialmente por años con cadencia decenal (1984, 1994, 2004, 2014). En consecuencia, el presente estudio se 
ha realizado sobre una base de 40 inventarios que han sido tratados mediante el "Método EFGMeaza" en su versión más evolucionada.

Los inventarios utilizados en el presente trabajo pueden consultarse a través del presente enlace: http://www.aranzadi.eus/fileadmin/docs/Munibe/InventariosHC_Online.pdf.

\section{Resultados de interactividad geoecológica registrados en cada etapa sucesional (por año de inventariado)}

Como puede apreciarse en la Tabla S1 (online) (http://www.aranzadi.eus/fileadmin/docs/Munibe/TablasSuplementariasOnlineS1-S4.pdf), las comunidades de fresnos de flor (Fraxinus ornus L.) o fresnales valencianos (Laguna et al., 2015b) estudiadas en 1984 presentan unos valores ciertamente bajos de fitoindicación, siendo especialmente llamativo el valor asociado a la actividad antrópica con un valor medio de 0,75. Es remarcable que en el conjunto de 10 parcelas inventariadas ese año, el resto de factores evidencien mayor importancia, casi el doble, en la delación de las especies que componen las poblaciones de esta comunidad vegetal. De hecho, el valor medio del rol fitoindicador para el factor antrópico muestra que es el más representativo del conjunto de factores, pues su desviación típica es la más baja $(0,22)$, a pesar de que en algunos inventarios su valor se sitúe por encima del de otros factores, caso de los inventarios $\mathrm{HC} 1$ y HC2. El resto de factores muestran valores ciertamente parecidos, aunque el mayor peso fitoindicador queda atribuido a los factores climático e hídrico (1,88 y 1,98 respectivamente).

En cuanto al rol fitoagente, destaca sobremanera la importancia de la influencia que estos fresnales ejercen sobre los factores: edáfico y antrópico, cuyas aportaciones a la funcionalidad geoecológica que explica el fenosistema de estas comunidades albergan valores muy superiores a los calculados para el resto de factores. Además, sus valores medios son indudablemente representativos del conjunto inventariado, pues su desviación típica es ciertamente baja -muy parecida a la del conjunto, salvo para el factor climático-. En este sentido, los valores más bajos en los 10 inventarios aparecen para el factor climático, con un valor medio de 0,72. A diferencia de lo analizado en el rol fitoindicador, el papel fitoagente respecto al factor que explica la disponibilidad de recursos para el ser humano (factor antrópico) gana peso específico en la configuración de este fenosistema, pues adquiere un valor de 1,39 y lo sitúa como el tercer factor de mayor impacto en la funcionalidad geoecológica de este fresnal en 1984.

En la Tabla 4, puede apreciarse la interactividad geoecológica expresada en el uso de los estadísticos explicados en la metodología. Se aprecia claramente un gradiente de interactividad positivo ajustado al nivel de complejidad estructural descrito (Laguna, 1997; Laguna et al. 2015b) -y que en este caso es aplicado el valor 3 (Meaza \& Cuesta, 2010; Gómez\& Meaza, 2010)en los factores edáfico $(0,26)$ y biótico $(0,32)$. No obstante, al establecer una tendencia futura mediante el empleo conjunto de valores estimados y sobreestimados se aprecia un incremento del gradiente de interactividad para los factores mencionados: edáfico $(0,45)$ y biótico $(0,49)$, así como la presencia de valores positivos para otros factores, en este caso, climático $(0,09)$ e hídrico $(0,16)$.

Las mismas parcelas estudiadas presentaban en 1994 un incremento del valor de rol bioindicador, siendo éste muy marcado en relación a las medias obtenidas para los factores climático, hídrico y geomorfológico; poco significativo en el resto de factores y prácticamente similar en la delación antrópica $(0,73)$. Consecuentemente, la diferencia entre los factores mencionados 


\begin{tabular}{|l|c|c|c|c|c|c|c|c|c|}
\hline \multirow{2}{*}{ K=3 } & \multicolumn{9}{|c|}{1984} \\
\cline { 2 - 10 } & \multicolumn{3}{|c|}{ TRATAMIENTO ESTADÍSTICO DE INTERACTIVIDAD FITOINDICACIÓN-FITOACCIÓN } \\
\hline FACTORES & Fitoind_s & Fitoacc_s & Ampl & $\begin{array}{l}\text { Grad_in } \\
\text { ter }\end{array}$ & $\begin{array}{l}\text { Fitoind_ } \\
\text { sobre st }\end{array}$ & $\begin{array}{l}\text { Fitoacc_ } \\
\text { sobre st }\end{array}$ & $\begin{array}{l}\text { Ampl__ } \\
\text { sobre st }\end{array}$ & $\begin{array}{l}\text { Grad_inter } \\
\text { sobre st }\end{array}$ & Ran_interact \\
\hline CLIMÁTICO & 1,88 & 0,72 & - & 0,00 & 2,19 & 1,07 & 0,27 & 0,09 & $0,00-0,09$ \\
\hline HÍDRICO & 1,98 & 1,00 & - & 0,00 & 2,28 & 1,21 & 0,49 & 0,16 & $0,00-0,16$ \\
\hline GEOMORFOLÓGICO & 1,24 & 1,05 & - & 0,00 & 1,50 & 1,19 & - & 0,00 & $0,00-0,00$ \\
\hline EDÁFICO & 1,55 & 2,25 & 0,80 & 0,26 & 1,92 & 2,42 & 1,34 & 0,45 & $0,26-0,45$ \\
\hline BIÓTICO & 1,44 & 2,51 & 0,95 & 0,32 & 1,70 & 2,76 & 1,46 & 0,49 & $0,32-0,49$ \\
\hline ANTRÓPICO & 0,75 & 1,39 & - & 0,00 & 0,98 & 1,53 & - & 0,00 & $0,00-0,00$ \\
\hline
\end{tabular}

Tabla 4.- Estadísticos obtenidos a partir de los datos de fitoindicación y fitoacción de 1984. Elaboración propia.

Table 4.- Phyto-indication and Phyto-action values calculated for the data set of 1984. Prepared by the authors.

respecto a la importancia del ser humano como condicionante de esta comunidad de fresno florido se incrementa hasta casi el triple en el caso de la fitoindicación hídrica $(2,27)$, siendo algo menor con respecto al factor climático $(2,16)$. En algunos inventarios, se aprecia muy claramente un marcado carácter condicionante del clima y la hidrología en el criptosistema que da lugar al paisaje percibido o fenosistema, son los casos de HC3, HC6 y, especialmente, HC4 (véase Tabla online S2).

En relación al carácter agente de la comunidad vegetal para influir en los factores de su hábitat, se sigue la tendencia de incremento de algunos factores que al igual que ocurría en los datos tomados en 1984, son principalmente los factores edáfico, biótico y antrópico. De éstos, destaca el valor de fitoacción biótica $(2,72)$ que incrementa su diferencia respecto al resto de valores medios obtenidos en relación a sus correspondientes valores obtenidos en 1984, mientras que el valor medio de fitoacción antrópica $(1,44)$ ve reducida su diferencia respecto a los valores obtenidos para los factores hídrico $(1,18)$ y geomorfológico $(1,12)$.

La interactividad geoecológica muestra una funcionalidad menos intensa que en 1984. Como puede apreciarse en la Tabla 5, el gradiente de interactividad vuelve a ser positivo para los factores edáfico y biótico pero con valores sustancialmente menores (0,25 y 0,31 respectivamente). Además, los datos sobreestimados también destilan una menor intensidad en el funcionamiento del criptosistema en comparación con la situación de 1984, pues tan solo aparecen tres factores con gradiente de interactividad positivo, caso de los factores hídrico, edáfico y biótico. En consecuencia, el rango de interactividad es sustancialmente menor que en 1984, no ya por el número de factores implicados, sino además, por los valores obtenidos en cada caso.

En 2004, comienza a vislumbrarse un incremento de algunos valores de fitoindicación que se habían mantenido poco representativos en las décadas anteriores. Es el caso de los factores biótico y edáfico, especialmente este último al superar el valor $2(2,13)$, confirmándose como el segundo factor cuya delación más ha crecido en la última década, solo por detrás del factor 


\begin{tabular}{|c|c|c|c|c|c|c|c|c|c|}
\hline \multirow{3}{*}{$\begin{array}{l}\mathrm{K}=4 \\
\text { FACTORES }\end{array}$} & \multicolumn{9}{|c|}{1994} \\
\hline & \multicolumn{9}{|c|}{ TRATAMIENTO ESTADÍSTICO DE INTERACTIVIDAD FITOINDICACIÓN-FITOACCIÓN } \\
\hline & Fitoind_s & Fitoacc_S & Ampl & $\begin{array}{l}\text { Grad_in } \\
\text { ter }\end{array}$ & $\begin{array}{l}\text { Fitoind_ } \\
\text { sobre st }\end{array}$ & $\begin{array}{l}\text { Fitoacc_ } \\
\text { sobre st }\end{array}$ & $\begin{array}{l}\text { Ampl_ } \\
\text { sobre st }\end{array}$ & $\begin{array}{l}\text { Grad_inter } \\
\text { _sobre st }\end{array}$ & Ran_interact \\
\hline CLIMÁTICO & 2,16 & 0,75 & - & 0,00 & 2,52 & 1,09 & - & 0,00 & $0,00-0,00$ \\
\hline HÍDRICO & 2,27 & 1,18 & - & 0,00 & 2,58 & 1,45 & 0,02 & 0,01 & $0,00-0,01$ \\
\hline GEOMORFOLÓGICO & 1,46 & 1,12 & - & 0,00 & 1,82 & 1,39 & - & 0,00 & $0,00-0,00$ \\
\hline EDÁFICO & 1,84 & 2,42 & 0,25 & 0,06 & 2,23 & 2,62 & 0,85 & 0,21 & $0,06-0,21$ \\
\hline BIÓTICO & 1,59 & 2,72 & 0,31 & 0,08 & 1,90 & 3,00 & 0,90 & 0,23 & $0,08-0,23$ \\
\hline ANTRÓPICO & 0,73 & 1,44 & - & 0,00 & 0,84 & 1,68 & - & 0,00 & $0,00-0,00$ \\
\hline
\end{tabular}

Tabla 5.- Estadísticos obtenidos a partir de los datos de fitoindicación y fitoacción de 1994. Elaboración propia.

Table 5.- Statistical values obtained from both phyto-indication and phyto-action data set of 1994. Prepared by the authors.

climático. El factor antrópico reduce bastante su importancia delatora, adquiriendo el valor más bajo del conjunto de factores $(0,53)$, siendo además significativamente más bajo que el obtenido en 1984 y 1994 (Tabla online S3).

En relación al rol fitoagente, cabe apreciar que la mayoría de factores, con excepción del biótico y del antrópico, ven paralizada la influencia que la comunidad ejerce en su control o condicionamiento con valores similares a los registrados en 1994. Tan solo se constata la influencia creciente que estos fresnales ejercen sobre los factores: biótico $(2,83)$ y antrópico $(1,56)$.

La interactividad geoecológica registrada en la Tabla 6 permite apreciar una funcionalidad todavía menos intensa que en 1984, atendiendo al número de factores con gradiente positivo,

\begin{tabular}{|l|c|c|c|c|c|c|c|c|c|}
\hline \multirow{2}{*}{ K=4 } & \multicolumn{8}{|c|}{2004} \\
\cline { 2 - 10 } & \multicolumn{3}{|c|}{ TRATAMIENTO ESTADÍSTICO DE INTERACTIVIDAD FITOINDICACIÓN-FITOACCIÓN } \\
\hline FACTORES & Fitoind_s & Fitoacc_S & Ampl & $\begin{array}{l}\text { Grad_in } \\
\text { ter }\end{array}$ & $\begin{array}{l}\text { Fitoind_ } \\
\text { sobre st }\end{array}$ & $\begin{array}{l}\text { Fitoacc_ } \\
\text { sobre st }\end{array}$ & $\begin{array}{l}\text { Ampl__ } \\
\text { sobre st }\end{array}$ & $\begin{array}{l}\text { Grad_inter } \\
\text { sobre st }\end{array}$ & Ran_interact \\
\hline CLIMÁTICO & 2,46 & 0,86 & - & 0,00 & 2,79 & 1,19 & - & 0,00 & $0,00-0,00$ \\
\hline HÍDRICO & 2,55 & 1,18 & - & 0,00 & 2,92 & 1,54 & 0,46 & 0,12 & $0,00-0,12$ \\
\hline GEOMORFOLÓGICO & 1,59 & 1,13 & - & 0,00 & 1,91 & 1,42 & - & 0,00 & $0,00-0,00$ \\
\hline EDÁFICO & 2,13 & 2,41 & 0,54 & 0,14 & 2,52 & 2,74 & 1,25 & 0,31 & $0,14-0,31$ \\
\hline BIÓTICO & 1,88 & 2,83 & 0,72 & 0,18 & 2,24 & 3,21 & 1,45 & 0,36 & $0,18-0,36$ \\
\hline ANTRÓPICO & 0,53 & 1,56 & - & 0,00 & 0,62 & 1,84 & - & 0,00 & $0,00-0,00$ \\
\hline
\end{tabular}

Tabla 6.- Estadísticos obtenidos a partir de los datos de fitoindicación y fitoacción de 2004. Elaboración propia.

Table 6.- Phyto-indication and Phyto-action values calculated for the data set of 2004. Prepared by the authors. 
pero con valores más altos que en 1994. Consecuentemente, considerando los valores obtenidos en el Rango de Interactividad, se puede apreciar un incremento de las interacciones, y por tanto de la funcionalidad, existente entre las poblaciones que componen la comunidad estudiada, funcionalidad geoecológica específicamente asociada a los factores hídrico, edáfico y biótico.

Como puede apreciarse en la Tabla S4 (online), la comunidad estudiada experimenta un incremento de la complejidad estructural y de madurez ecológica (Laguna et al., 2015b) al final de la serie temporal analizada -2014-, hecho que permite emplear la constante K=5 (Meaza \& Cuesta, 2010) para el cálculo de su funcionalidad geoecológica. Consecuentemente, se aprecia un incremento notable de la influencia de todos los factores mesológicos estudiados, con excepción del factor antrópico, en la caracterización estructural y funcional de esta comunidad dominada por fresno florido, donde sigue siendo destacable la influencia del factor hídrico $(3,52)$ junto al factor climático $(3,38)$. Los factores geomorfológico, edáfico y biótico, también han incrementado su influencia en la organización ecológica de estas comunidades a la par que éstas han ido progresando en la sucesión vegetal descrita, adquiriendo valores de 2,28, 2,98 y 2,79 respectivamente. De igual manera, se mantiene la tendencia seguida por el factor antópico durante toda la secuencia temporal descrita, siendo ésta casi testimonial en la comunidad estudiada. El resultado obtenido para este factor $(0,70)$, no solo es más bajo que el obtenido en los años 1984 y 1994, a pesar de haber ido incrementándose el valor de la constante de complejidad estructural (K) hasta el valor máximo adjudicado por la presente propuesta metodológica, sino que además se ha incrementado la diferencia respecto a los valores obtenidos para el resto de factores mesológicos.

En el caso de la influencia que ejerce la propia comunidad sobre el conjunto de factores mesológicos agrupados en la descripción sintética de su hábitat, se aprecia que se sigue la tendencia iniciada en 1984, siendo los factores más condicionados por la actividad geoecológica de estos fenosistemas, el factor biótico $(4,02)$, seguido por los factores: edáfico $(3,23)$ y antrópico $(2,20)$. El resto de factores condicionados por estas comunidades también incrementan sus valores, especialmente los factores: hídrico $(1,72)$ y geomorfológico $(1,57)$. Cabe recordar que se trata de comunidades ubicadas en laderas y fondos de valle, donde su interacción con estos factores coadyuvan a la estabilidad de las vertientes y sus suelos.

La funcionalidad geoecológica, determinada en esta metodología como interactividad entre las funciones delatoras y controladoras de los factores del medio (Meaza \& Cuesta, 2010), vuelve a intensificarse en 2014, como puede apreciarse en la Tabla 7. En efecto, no solo se incrementa el número de factores implicados, sino que los que se habían mantenido en la etapa sucesional anterior -determinada por la constante de complejidad estructural K=4-, también ven incrementado sus correspondientes valores, tanto en los determinados por el valor medio, como en los valores sobreestimados en los que también se tiene en cuenta la desviación típica para su cálculo. En consecuencia, se aprecia un Rango de interactividad positivo en todos los factores excepto para los agrupados en las categorías: geomorfológico y antrópico.

\section{Confrontación de resultados de interactividad geoecológica registrados en cada etapa sucesional}

Los resultados obtenidos delatan una complejidad creciente en el reajuste de interacciones y 


\begin{tabular}{|c|c|c|c|c|c|c|c|c|c|}
\hline \multirow{3}{*}{$\begin{array}{l}\mathrm{K}=5 \\
\text { FACTORES }\end{array}$} & \multicolumn{9}{|c|}{2014} \\
\hline & \multicolumn{9}{|c|}{ TRATAMIENTO ESTADÍSTICO DE INTERACTIVIDAD FITOINDICACIÓN-FITOACCIÓN } \\
\hline & Fitoind_s & Fitoacc_s & Ampl & $\begin{array}{l}\text { Grad_in } \\
\text { ter }\end{array}$ & $\begin{array}{l}\text { Fitoind_ } \\
\text { sobre st }\end{array}$ & $\begin{array}{l}\text { Fitoacc_ } \\
\text { sobre st }\end{array}$ & $\begin{array}{l}\text { Ampl_ } \\
\text { sobre st }\end{array}$ & $\begin{array}{l}\text { Grad_inter } \\
\text { _sobre st }\end{array}$ & Ran_interact \\
\hline CLIMÁTICO & 3,38 & 1,43 & - & 0,00 & 3,98 & 1,82 & 0,80 & 0,16 & $0,00-0,16$ \\
\hline HÍDRICO & 3,52 & 1,72 & 0,25 & 0,05 & 4,23 & 2,18 & 1,41 & 0,28 & $0,05-0,28$ \\
\hline GEOMORFOLÓGICO & 2,28 & 1,57 & - & 0,00 & 2,73 & 1,96 & - & 0,00 & $0,00-0,00$ \\
\hline EDÁFICO & 2,98 & 3,23 & 1,21 & 0,24 & 3,46 & 3,97 & 2,43 & 0,49 & $0,24-0,49$ \\
\hline BIÓTICO & 2,79 & 4,02 & 1,81 & 0,36 & 3,30 & 4,77 & 3,08 & 0,62 & $0,36-0,62$ \\
\hline ANTRÓPICO & 0,70 & 2,20 & - & 0,00 & 0,86 & 2,73 & - & 0,00 & $0,00-0,00$ \\
\hline
\end{tabular}

Tabla 7.- Estadísticos obtenidos a partir de los datos de fitoindicación y fitoacción de 2014. Elaboración propia.

Table 7.- Statistical values obtained from both phyto-indication and phyto-action data set of 2014. Prepared by the authors.

su correspondiente intensidad conforme la sucesión vegetal se traduce en una evolución hacia etapas ecológicamente más maduras. En este sentido, se ha comparado el papel que juega cada factor en la funcionalidad geoecológica durante la sucesión, tanto en su papel delator, ejercido por las especies que componen estas comunidades, como en el rol condicionante que éstas ejercen en el conjunto de factores mesológicos que determinan su hábitat.

Para ello se ha utilizado en primer lugar el test de significación estadística $t$ de Student, lo que ha permitido confrontar los valores medios obtenidos para cada factor en cada unidad muestreada en los diferentes años en los que se basa este estudio. En tal sentido, se ha procedido a confrontar los valores de 1984 respecto a 1994, 1994 respecto a 2004, 2004 con 2014, pudiéndose comprobar los resultados en la Tabla 8.

Los resultados obtenidos revelan que en la gran mayoría de transiciones entre muestreos decenales sucesivos -de 1984 a 1994, de 1994 a 2004 y de 2004 a 2014- existen diferencias significativas entre los valores medios de las situaciones inicial y final. Estos cambios son más aparentes para los valores de la fitoindicación climática, hídrica, edáfica y biótica, donde todas las transiciones arrojan valores significativos y del mismo signo -creciente en todas excepto en la fitoindicación biótica, donde el valor tiende a reducirse al avanzar la evolución de la vegetación. Cabe destacar que en todos los casos, tanto de fitoindicación como de fitoacción, es significativa la diferencia de las medias entre los valores de 2004 y 2014. Esta significación coincide con el momento en que, conforme a los resultados expresados por Laguna et al. (2015a), se produce una reducción más drástica de la primitiva dominancia de la cobertura de las coníferas -Pinus halepensis y P. pinaster subsp. escarena-, al tiempo que se disparan la de las frondosas climácicas -Quercus faginea y Q. ilex subsp. rotundifolia-. En consecuencia, los valores de fitoindicación y fitoacción servirían para corroborar los cambios en el ecosistema en ese punto de inflexión.

El análisis estadístico se completa con una aproximación al carácter fitoindicador o fitoagente de la comunidad vegetal en cada año, empleando para ello de nuevo el test $t$ de Student a la 


\begin{tabular}{|c|c|c|c|c|c|c|c|}
\hline \multicolumn{5}{|c|}{ AÑOS } & \multicolumn{3}{|c|}{ TRANSICCIONES DECENALES } \\
\hline & 1984 & 1994 & 2004 & 2014 & 1994-1984 & 2004-1994 & $2014-2004$ \\
\hline $\mathrm{Fi} C l i$ & $1,88+/-0,31$ & $2,16+/-0,36$ & $2,46+/-0,33$ & $3,38+/-0,59$ & $0,28+/-0,26$ & $0,30+/-0,26$ & $0,93+/-0,61$ \\
\hline$t(p)$ & & & & & $3,330(0,009)$ & $3,535(0,006)$ & $4,811(0,001)$ \\
\hline $\mathrm{FaCli}$ & $0,72+/-0,36$ & $0,75+/-0,33$ & $0,86+/-0,33$ & $1,43+/-0,40$ & $0,03+/-0,21$ & $0,10+/-0,23$ & $0,57+/-0,24$ \\
\hline$t(p)$ & & & & & $0,504(0,627 \mathrm{~ns})$ & $1,400(0,195 \mathrm{~ns})$ & $7,419(<0,0001)$ \\
\hline Fi Hid & $1,98+/-0,31$ & $2,27+/-0,31$ & $2,55+/-0,37$ & $3,52+/-0,71$ & $0,29+/-0,26$ & $0,28+/-0,26$ & $0,97+/-0,67$ \\
\hline$t(p)$ & & & & & $3,537(0,006)$ & $3,429(0,008)$ & $4,553(0,001)$ \\
\hline Fa Hid & $1,00+/-0,21$ & $1,18+/-0,26$ & $1,18+/-0,36$ & $1,72+/-0,46$ & $0,19+/-0,26$ & $-0,01+/-0,15$ & $0,55+/-0,22$ \\
\hline$t(p)$ & & & & & $2,289(0,048)$ & $-0,175(0,865 \mathrm{~ns})$ & $7,748(<0,0001)$ \\
\hline $\mathrm{Fi} \mathrm{Geo}$ & $1,24+/-0,26$ & $1,46+/-0,36$ & $1,59+/-0,31$ & $2,28+/-0,45$ & $0,22+/-0,23$ & $0,14+/-0,25$ & $0,69+/-0,38$ \\
\hline$t(p)$ & & & & & $3,041(0,014)$ & $1,745(0,115 \mathrm{~ns})$ & $5,798(0,0003)$ \\
\hline $\mathrm{Fa} \mathrm{GeO}$ & $1,05+/-0,14$ & $1,12+/-0,26$ & $1,13+/-0,30$ & $1,57+/-0,39$ & $0,07+/-0,20$ & $0,00+/-0,16$ & $0,44+/-0,27$ \\
\hline$t(p)$ & & & & & $1,127(0,289 \mathrm{~ns})$ & $0,034(0,973$ ns $)$ & $5,088(0,001)$ \\
\hline Fi Eda & $1,55+/-0,37$ & $1,84+/-0,39$ & $2,13+/-0,39$ & $2,98+/-0,47$ & $0,28+/-0,19$ & $0,29+/-0,25$ & $0,86+/-0,49$ \\
\hline$t(p)$ & & & & & $4,727(0,001)$ & $3,773(0,004)$ & $5,482(0,0004)$ \\
\hline Fa Eda & $2,25+/-0,17$ & $2,42+/-0,21$ & $2,41+/-0,33$ & $3,23+/-0,75$ & $0,17+/-0,18$ & $-0,01+/-0,22$ & $0,82+/-0,66$ \\
\hline$t(p)$ & & & & & $2,882(0,018)$ & $-0,101(0,922 \mathrm{~ns})$ & $3,938(0,003)$ \\
\hline Fi Bio & $2,79+/-0,51$ & $1,88+/-0,36$ & $1,59+/-0,31$ & $1,44+/-0,26$ & $-0,91+/-0,45$ & $-0,30+/-0,27$ & $-0,15+/-0,15$ \\
\hline$t(p)$ & & & & & $-3,148(0,012)$ & $-3,499(0,007)$ & $-3,148(0,012)$ \\
\hline Fa Bio & $2,51+/-0,24$ & $2,72+/-0,28$ & $2,83+/-0,38$ & $4,02+/-0,75$ & $0,21+/-0,33$ & $0,11+/-0,37$ & $1,19+/-0,72$ \\
\hline$t(p)$ & & & & & 1,985 (0,078 ns) & $0,947(0,368 \mathrm{~ns})$ & $5,195(0,001)$ \\
\hline Fi Ant & $0,75+/-0,22$ & $0,73+/-0,12$ & $0,53+/-0,09$ & $0,70+/-0,16$ & $-0,02+/-0,20$ & $-0,20+/-0,13$ & $0,18+/-0,18$ \\
\hline$t(p)$ & & & & & $-0,361(0,727 \mathrm{~ns})$ & $-4,491(0,001)$ & $3,081(0,013)$ \\
\hline Fa Ant & $1,39+/-0,15$ & $1,44+/-0,24$ & $1,56+/-0,28$ & $2,20+/-0,53$ & $0,05+/-0,21$ & $0,12+/-0,25$ & $0,64+/-0,37$ \\
\hline$t(p)$ & & & & & $0,760(0,467 \mathrm{~ns})$ & $1,483(0,172 \mathrm{~ns})$ & $5,484(0,0003)$ \\
\hline
\end{tabular}

combinación entre Fi y Fa - diferencia entre los valores de fitoacción respecto a fitoindicacióny con la comparación completa de todas las muestras de un mismo factor mediante ANOVA simple, obteniendo tanto los valores para todo el recorrido temporal, como los de cada par de muestras -años- a través del estadístico Q de Tukey.

La aplicación del test $t$ de Student a la combinación Fi y Fa pretende estimar, junto a otros elementos de análisis, hasta qué punto la vegetación actúa más como fitoagente que como fi- 
Tabla 8.- Valores medios y desviaciones estándar de los 10 inventarios de vegetación en los 4 años de muestreo, así como de las transiciones decenales - diferencias entre los valores de años sucesivos de inventariado, restando el más antiguo al más reciente-, para las 6 variables consideradas de Fitoindicación (Fi) y Fitoacción ( $\mathrm{Fa}$ ). Las transiciones corresponde a la resta de los valores entre el año más reciente y el anterior más antiguo de muestreo en cada inventario. Bajo cada media y desviación de las transiciones se adjunta el valor del estadístico t de Student para testar la diferencia de las medias de datos apareados, con su correspondiente probabilidad (p). En itálicas, comparaciones en las que se observa diferencia significativa de las muestras para $\mathrm{p}=0,05$ o inferior; ns: diferencia no significativa. Notación de las variables: Cli: Climática; Hid: Hídrica; Geo: Geomorfológica; Eda: Edáfica; Bio: Biótica; Ant: Antrópica.

Table 8.- Mean values and standard deviations for 10 samples in each of the four years of sampling, and the decennial transitions - difference between consecutive years of sampling, subtracting the old one from the most recent one-, for the 6 variables considered of phyto-indication (Fi) and phytoaction (Fa). Below each average and transition values, $t$ Student values can be regarded to test the difference of the calculated averages from two-paired data with their pertinent probability (p). Those figures with significant differences for $p=0,05$ or less are shown in italics; ns: no difference. Variable notation: Cli: climate; Hid: Hydrology; Geo: Geomorphology; Eda: Soils; Bio: Biota; Ant: Human.

toindicador o viceversa, permitiendo obtener una visión sobre cómo se combinan esos dos valores característicos de la comunidad vegetal en ese momento. Una diferencia positiva nos indica que la comunidad vegetal tiene mucho más valor como fitoagente que como fitoindicador, y en tanto se incremente dicho valor, cabe prever que ese tipo de vegetación está aumentando su efecto de interacción con el medio (p.ej. aumentando su capacidad de fijación del suelo, generando una mayor acogida de fauna silvestre, etc.). Los valores negativos no implican una pérdida de calidad del hábitat, sino la preponderancia de la información que la unidad de vegetación nos aporta a través de su papel como fitoindicador, respecto a la de su acción como elemento básico y a la vez transformador del propio ecosistema.

Al estudiar cómo varían en el tiempo los valores del parámetro Fa - Fi (Tabla 9) se observa que existen diferencias significativas de las medias entre años sucesivos para todas las transiciones de las variables climática y geomorfológica, y en ambos casos son siempre de signo negativo. Ello implica que a medida que avanza la sucesión vegetal dentro de los fresnales, pasa a tener más importancia la función explicativa o indicadora del ecosistema que tienen las especies de la comunidad vegetal, frente a la del papel fitoagente. Este resultado es lógico, ya que estas dos variables son precisamente aquellas en las que el medio tiende a condicionar más a la vegetación, y donde ésta, a su vez, puede modificar menos o a menor velocidad los condicionantes ambientales -en este caso el clima y las grandes directrices del relieve-. El signo de las transiciones sólo tiende a ser positivo -aunque no con diferencias significativas en todas ellapara las variables: biótica y antrópica, lo que nuevamente puede asociarse con explicaciones lógicas sobre el funcionalismo de los fresnales. De un lado, es evidente que el incremento en la sucesión hacia una posible etapa climácica final implica un incremento progresivo de la complejidad funcional y diversidad del ecosistema, lo que necesariamente ha de revertir en un aumento del rol fitoagente -p.ej. a través del aumento cuantitativo y cualitativo de especies de fauna hospedadas por la comunidad, la aparición de nuevas relaciones de mutualismo, etc. Por otro, ese mismo incremento de funciones y especies da lugar a un aumento de los servicios ecosistémicos y en particular de los que producen más beneficio tangible al ser humano -p.ej. 


\begin{tabular}{|c|c|c|c|c|c|c|c|}
\hline \multicolumn{5}{|c|}{ AÑOS } & \multicolumn{3}{|c|}{ TRANSICCIONES DECENALES } \\
\hline & 1984 & 1994 & 2004 & 2014 & 1994-1984 & 2004-1994 & $2014-2004$ \\
\hline $\begin{array}{l}\mathrm{Fa}-\mathrm{Fi} \\
\mathrm{Cli}\end{array}$ & $-1,16+/-0,39$ & $-1,41+/-0,25$ & $-1,60+/-0,22$ & $-1,96+/-0,32$ & $-0,24+/-0,31$ & $-0,19+/-0,14$ & $-0,36+/-0,45$ \\
\hline$t(p)$ & & & & & $-2,488(0,035)$ & $-4,222(0,002)$ & $-2,514(0,033)$ \\
\hline $\begin{array}{l}\mathrm{Fa}-\mathrm{Fi} \\
\mathrm{Hid}\end{array}$ & $-0,98+/-0,48$ & $-1,08+/-0,26$ & $-1,37+/-0,28$ & $-1,80+/-0,31$ & $-0,10+/-0,37$ & $-0,29+/-0,21$ & $-0,42+/-0,51$ \\
\hline$t(p)$ & & & & & $-0,902(0,390 \mathrm{~ns})$ & $-4,451(0,002)$ & $-2,631(0,027)$ \\
\hline $\begin{array}{l}\mathrm{Fa}-\mathrm{Fi} \\
\mathrm{GeO}\end{array}$ & $-0,19+/-0,26$ & $-0,33+/-0,23$ & $-0,47+/-0,20$ & $-0,72+/-0,18$ & $-0,14+/-0,18$ & $-0,14+/-0,17$ & $-0,25+/-0,18$ \\
\hline$t(p)$ & & & & & $-2,616(0,028)$ & $-2,485(0,035)$ & $-4,460(0,002)$ \\
\hline $\begin{array}{l}\mathrm{Fa}-\mathrm{Fi} \\
\mathrm{Eda}\end{array}$ & $0,70+/-0,47$ & $0,58+/-0,41$ & $0,28+/-0,31$ & $0,25+/-0,43$ & $-0,11+/-0,17$ & $-0,30+/-0,21$ & $-0,04+/-0,26$ \\
\hline$t(p)$ & & & & & $-2,196(0.056 \mathrm{~ns})$ & $-4,567(0,001)$ & $-0,436(0,673 \mathrm{~ns})$ \\
\hline $\begin{array}{l}\mathrm{Fa}-\mathrm{Fi} \\
\mathrm{BiO}\end{array}$ & $-0,28+/-0,63$ & $0,84+/-0,32$ & $1,24+/-0,34$ & $2,58+/-0,79$ & $1,11+/-0,63$ & $0,41+/-0,62$ & $1,33+/-0,77$ \\
\hline$t(p)$ & & & & & $5,601(0,0003)$ & $2,078(0,067 \mathrm{~ns})$ & $5,453(0,0004)$ \\
\hline $\begin{array}{l}\mathrm{Fa}-\mathrm{Fi} \\
\text { Ant }\end{array}$ & $0,64+/-0,20$ & $0,71+/-0,30$ & $1,03+/-0,30$ & $1,50+/-0,42$ & $0,07+/-0,04$ & $0,32+/-0,35$ & $0,47+/-0,43$ \\
\hline$t(p)$ & & & & & $0,871(0,406 \mathrm{~ns})$ & $4,373(0,002)$ & $6,155(0,0002)$ \\
\hline
\end{tabular}

Tabla 9.- Medias y desviaciones estándar obtenidos cada año de muestreo para el valor de la interactividad no corregida -sin aplicación del coeficiente K-, obtenido al restar el valor de Fitoindicación (Fi) al de Fitoacción ( $\mathrm{Fa}$ ) en cada inventario, para las 6 variables consideradas. A la derecha, medias y desviaciones obtenidas en las transiciones decenales de $\mathrm{Fa}-\mathrm{Fi}$, valores del estadístico t para el testado de diferencias de las medias de datos apareados, y de su correspondiente probabilidad.

Table 9.- Mean values and standard deviations obtained for values from each year without $\mathrm{K}$ coefficient. The Phyto-indication (Fi) value has been subtracted from the Phyto-action (Fa) value in each sample for the 6 variables considered. To the right, averages and standard deviations obtained through decennial transitions (Fa-Fi), value of t Student to test differences between averages of twopaired data and their inherent probability.

aumento del volumen de madera, incremento del número de especies de interés apícola, mayor valor estético y de usos recreativos, etc.

El análisis ANOVA simple se ha completado con las pruebas de Levene de homogeneidad de las varianzas y el test $F$ de Welch para la hipótesis de disimilaridad de al menos una de las varianzas (Tabla 10).

Al aplicar el análisis de la varianza a la diferencia entre los valores de fitoindicación y fitoacción para cada una de las 6 variables mesológicas, comparando los valores de los 4 años de muestreo, se observan resultados significativos para todas las variables, es decir, que en cada una de ellas puede aceptarse que las medias varían entre los años en que se han realizado los inventarios. Esta diferencia sólo se acerca el valor crítico de falta de significación $(p=0,05)$ en el caso de la variable edáfica $(p=0,0444)$; de hecho, aplicando el test $F$ de Welch si hubiéramos partido de la hipótesis de que al menos alguna de las varianzas fuera significativamente diferente -el test de Levene corrobora, no obstante, que sí que son similares, al valor obtenido sería de $p=0,0731$. El único caso en que se observa claramente disimilaridad de las varianzas es en el 


\begin{tabular}{|c|c|c|c|c|c|c|}
\hline & Climática & Hídrica & Geomorfológica & Edáfica & Biótica & Antrópica \\
\hline$F_{3,36}(p)$ & $\begin{array}{c}12,32 \\
(1,07 E-05)\end{array}$ & $\begin{array}{c}11,32 \\
(2,23 E-05)\end{array}$ & $\begin{array}{c}10,44 \\
(4,38 \mathrm{E}-05)\end{array}$ & $\begin{array}{c}2,97 \\
(0,0444)\end{array}$ & $\begin{array}{c}45,03 \\
(2,85 E-12)\end{array}$ & $\begin{array}{c}15,43 \\
(1,29 \mathrm{E}-06)\end{array}$ \\
\hline$p$ Levene & 0,2198 ns & $0,1908 \mathrm{~ns}$ & $0,7069 \mathrm{~ns}$ & 0,3924 ns & 0,0014 & 0,2503 ns \\
\hline$p$ Levene & 0,3734 ns & 0,2017 ns & 0,6934 ns & $0,4855 \mathrm{~ns}$ & 0,0049 & 0,2783 ns \\
\hline Welch F $(p)$ & $\begin{array}{c}9,39 \\
(0,0005)\end{array}$ & $\begin{array}{c}11,54 \\
(0,0001)\end{array}$ & $\begin{array}{c}10,64 \\
(0,0002)\end{array}$ & $\begin{array}{c}2,71 \\
(0,0731 \mathrm{~ns})\end{array}$ & $\begin{array}{c}27,73 \\
(3,60 \mathrm{E}-07)\end{array}$ & $\begin{array}{c}12,53 \\
(8,79 E-05)\end{array}$ \\
\hline 1984 vs 1994 & $\begin{array}{c}2,549 \\
(0,2889 \mathrm{~ns})\end{array}$ & $\begin{array}{c}0,965 \\
(0,9032 \text { ns })\end{array}$ & $\begin{array}{c}2,102 \\
(0,4559 \text { ns })\end{array}$ & $\begin{array}{c}0,898 \\
(0,9201 \mathrm{~ns})\end{array}$ & $\begin{array}{c}6,342 \\
(0,0005)\end{array}$ & $\begin{array}{c}0,743 \\
(0,9525 n s)\end{array}$ \\
\hline 1984 vs 2004 & $\begin{array}{c}4,555 \\
(0,0139)\end{array}$ & $\begin{array}{c}3,640 \\
(0,0655 \text { ns })\end{array}$ & $\begin{array}{c}4,046 \\
(0,0338\end{array}$ & $\begin{array}{c}3,205 \\
(0,1253 \text { ns })\end{array}$ & $\begin{array}{c}8,657 \\
(0,0002)\end{array}$ & $\begin{array}{c}3,965 \\
(0,0388)\end{array}$ \\
\hline 1984 vs 2014 & $\begin{array}{c}8,316 \\
(0,0002)\end{array}$ & $\begin{array}{c}7,519 \\
(0,0002)\end{array}$ & $\begin{array}{c}7,603 \\
(0,0002)\end{array}$ & $\begin{array}{c}3,514 \\
(0,0796 \mathrm{~ns})\end{array}$ & $\begin{array}{c}15,250 \\
(0,0002)\end{array}$ & $\begin{array}{c}8,632 \\
(0,0002)\end{array}$ \\
\hline 1994 vs 2004 & $\begin{array}{c}2,006 \\
(0,4965 \text { ns })\end{array}$ & $\begin{array}{c}2,675 \\
(0,2497 \text { ns })\end{array}$ & $\begin{array}{c}1,944 \\
(0,5231 \text { ns })\end{array}$ & $\begin{array}{c}2,307 \\
(0,3747 \mathrm{~ns})\end{array}$ & $\begin{array}{c}2,315 \\
(0,3715 \mathrm{~ns})\end{array}$ & $\begin{array}{c}3,222 \\
(0,1223 \text { ns })\end{array}$ \\
\hline 1994 vs 2014 & $\begin{array}{c}5,767 \\
(0,0014)\end{array}$ & $\begin{array}{c}6,554 \\
(0,0004)\end{array}$ & $\begin{array}{c}5,501 \\
(0,0024)\end{array}$ & $\begin{array}{c}2,616 \\
(0,2675 \text { ns })\end{array}$ & $\begin{array}{c}9,908 \\
(0,0002)\end{array}$ & $\begin{array}{c}7,889 \\
(0,0002)\end{array}$ \\
\hline 2004 vs 2014 & $\begin{array}{c}3,761 \\
(0,0541 \text { ns })\end{array}$ & $\begin{array}{c}3,879 \\
(0,0447)\end{array}$ & $\begin{array}{c}3,557 \\
(0,0746 \text { ns })\end{array}$ & $\begin{array}{c}0,310 \\
(0,9963 \mathrm{~ns})\end{array}$ & $\begin{array}{c}7,593 \\
(0,0002)\end{array}$ & $\begin{array}{c}4,667 \\
(0,0113)\end{array}$ \\
\hline
\end{tabular}

Tabla 10.- Resultado de la aplicación del ANOVA simple a los valores de interactividad (Fa - Fi de cada inventario y año) comparando los 4 años de muestreo. Además de los valores de $F$ y $p$, para la diferencia de las muestras, se expresan los de los tests apareados (Tukey), la probabilidad ( $p$ ) en las pruebas de Levene para el testado de la homogeneidad de las varianzas y el test $F$ de Welch para la diferencia de las muestras bajo la hipótesis de varianzas desiguales.

Table 10.- Result of ANOVA for interactivity values (Fa-Fi of each year) comparing all sampling dates. Besides values of $F$ and $p$, for the difference of samples expressed by two-paired test (Tukey), probability $(p)$ in Levene test to know the homogeneity of variances and the $F$ test of Welch for the difference between samples under the unequal variances hypothesis.

del factor biótico - $p$ de la prueba de Levene para medias y medianas de 0,0014 y 0,0049 respectivamente-, pero incluso en esa situación el test de Welch obliga a considerar que existe una clara diferencia entre los valores anuales analizados.

\section{Discusión}

Los resultados obtenidos a partir del tratamiento de estos datos con el "Método EFG-Meaza" permiten conocer qué factores - y su grado de incidencia- han gozado de mayor influencia en la dinámica sucesional descrita por el trabajo de Laguna et al. (2015b), evidenciando que la 
evolución de la estructura y composición de esta comunidad vegetal no sigue una pauta continuada de intensidad en su funcionalidad geoecológica, sino que estas interacciones albergan un comportamiento ciertamente complejo que puede ser asociado a procesos analizados a esta escala de análisis propuesta, pero también a otros procesos, en principio poco conocidos o poco evidentes siguiendo esta propuesta metodológica.

En este sentido, aspectos como la escasa aportación del factor antrópico como elemento que puede influir en la composición y estructura de la comunidad estudiada se explica perfectamente por el grado de protección adquirido por el territorio estudiado, no en vano a principios del siglo XX la sierra valenciana de Malacara se encontraba fuertemente desertificada como consecuencia de la sobreexplotación de sus recursos, por lo que la Administración de la época tomó una serie de medidas que incluían la repoblación, su incorporación al Catálogo de Montes de Utilidad Pública, las limitaciones de pastoreo y de extracción de madera (Laguna, 1995; Laguna et al., 2015b). Además, en el caso de este factor cabe indicar su fuerte contraste con su rol fitoagente, es decir en como el fresnal puede influir en las actividades humanas, siendo éste ciertamente intenso pues se trata de una comunidad integrada por especies que podrían ser utilizadas de manera sostenible por el ser humano y que, dadas las restricciones legales existentes, sus recursos no están siendo explotados como claramente refleja la fitoindicación antrópica.

Tal y como se ha insinuado previamente, la funcionalidad geoecológica analizada no sigue un patrón lineal de progresión de la intensidad, por ejemplo puede observarse una reducción de la intensidad en la funcionalidad geocológica entre 1984 y 1994, que claramente se asocia al incremento de la complejidad estructural. En efecto, entre 1984 y 1994 se produce un incremento de la complejidad estructural de la comunidad estudiada, reduciéndose el número de táxones en algunos estratos e incrementándose en otros, pero sobre todo adquiriendo el nivel estructural denominado por la metodología empleada como tipo 4 (Meaza \& Cuesta, 2010), cabe recordar que la comunidad estudiada se encontraba en tipo 3 en 1984. En este sentido, la explicación cabe buscarla en que la comunidad necesita un tiempo de reajuste en sus relaciones ecológicas cuando se produce un incremento de la presencia de una especie o un aumento de su representatividad, que en este caso, además, se asocia a un importante cambio estructural. Por consiguiente, el criptosistema debe acomodarse a la nueva situación alcanzada por su fenosistema.

No obstante, a pesar del aparentemente errático comportamiento de las interacciones geoecológicas durante la sucesión estudiada para estos fresnales valencianos, esta metodología permite comprobar el grado de complejidad creciente de las relaciones ecológicas que englobadas en el criptosistema determinan el paisaje vegetal analizado a partir de la caracterización de su fenosistema (Fig. 4). Se aprecia una clara evolución de la funcionalidad geoecológica conforme se avanza temporalmente hacia etapas más maduras, vislumbrándose además en el gráfico de 2014 qué factores van a incrementar su protagonismo en un futuro inmediato. En consecuencia, esta metodología permite modelizar sobre la influencia de los diferentes factores del medio que coadyuvarán en el fenosistema de fresnal que podrá apreciarse entonces. Por último, el presente trabajo pone de manifiesto que la metodología empleada ("Método EFG-Meaza") es una herramienta muy útil de cara a analizar detalladamente las diferentes etapas de la dinámica vegetal (sucesión temporal) al aplicarse exitosamente a un inventariado diacró- 


\section{4}

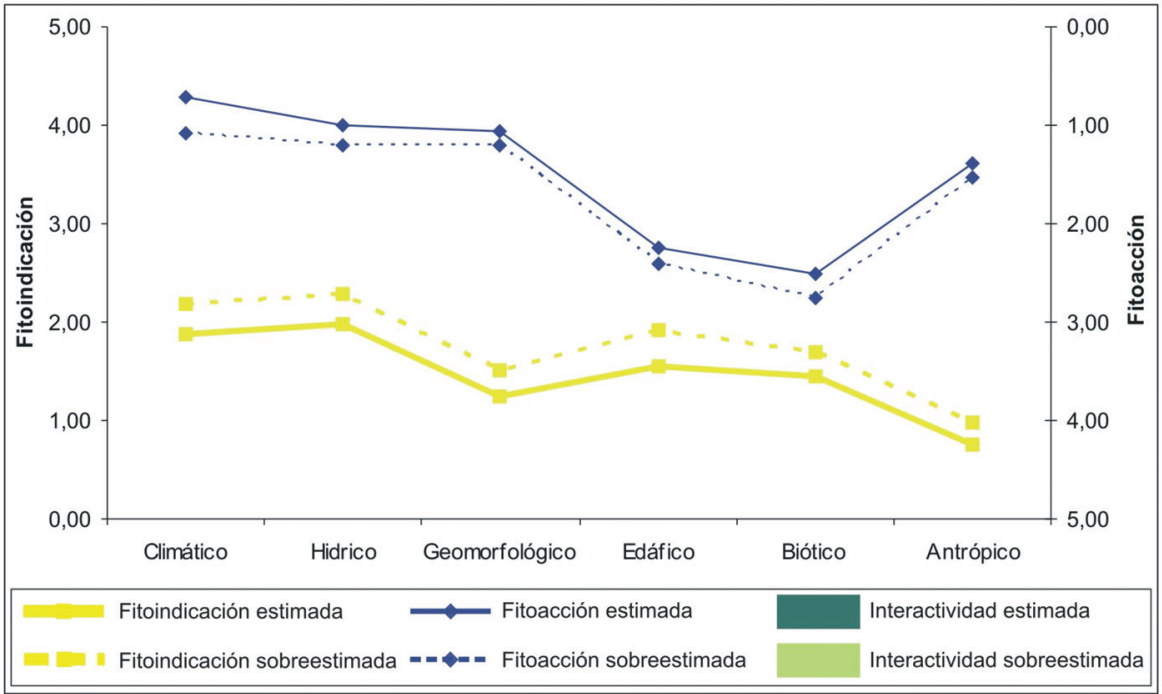

\section{4}

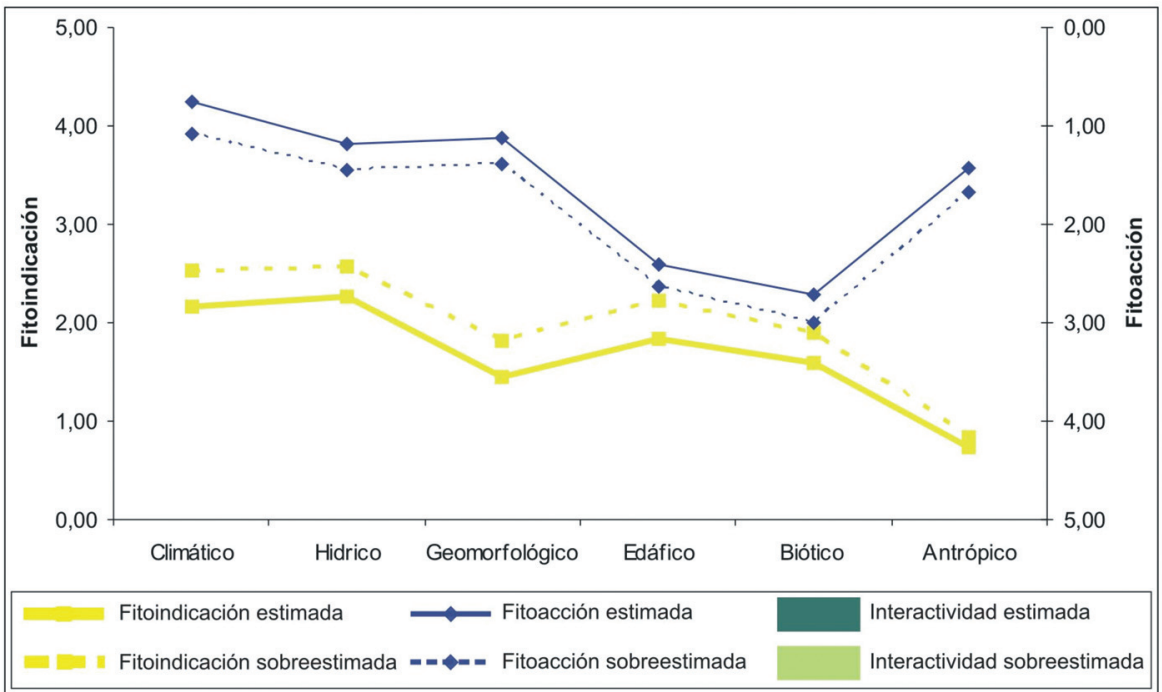

Fig. 4.- Interactividad fitoindicación-fitoacción en los diferentes muestreos con cadencia decenal. Elaboración propia.

Fig. 4.- Phyto-indication and Phyto-action interactivity in each decennial data set. Prepared by the authors. 


\section{4}

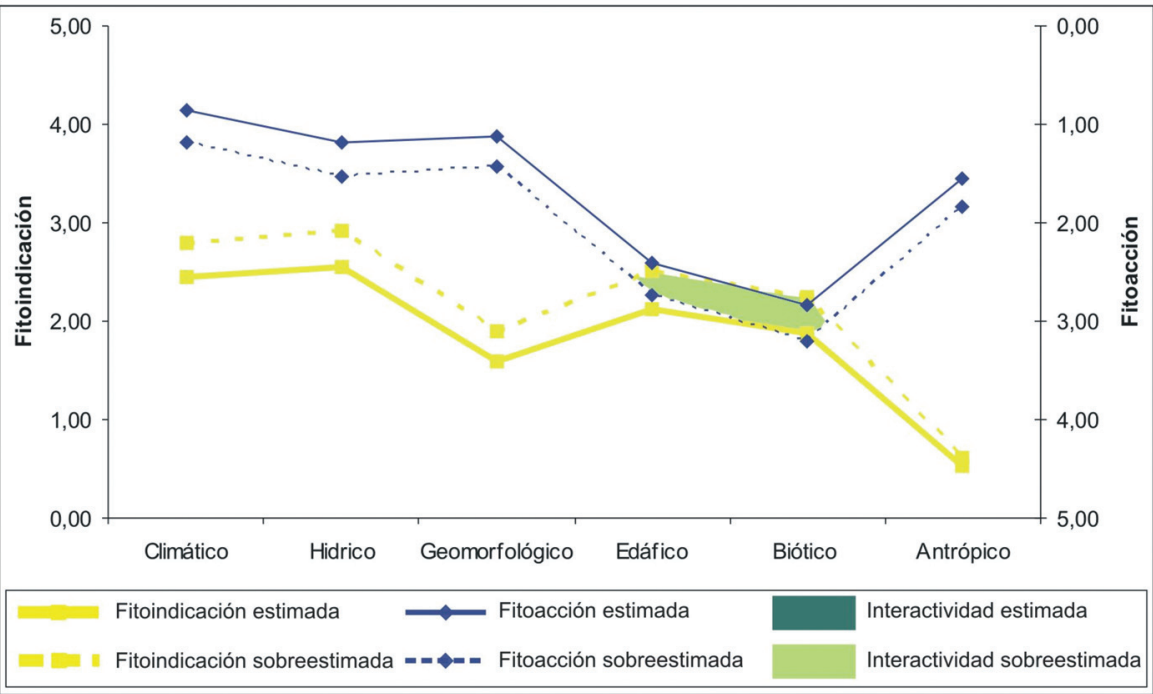

\section{4}

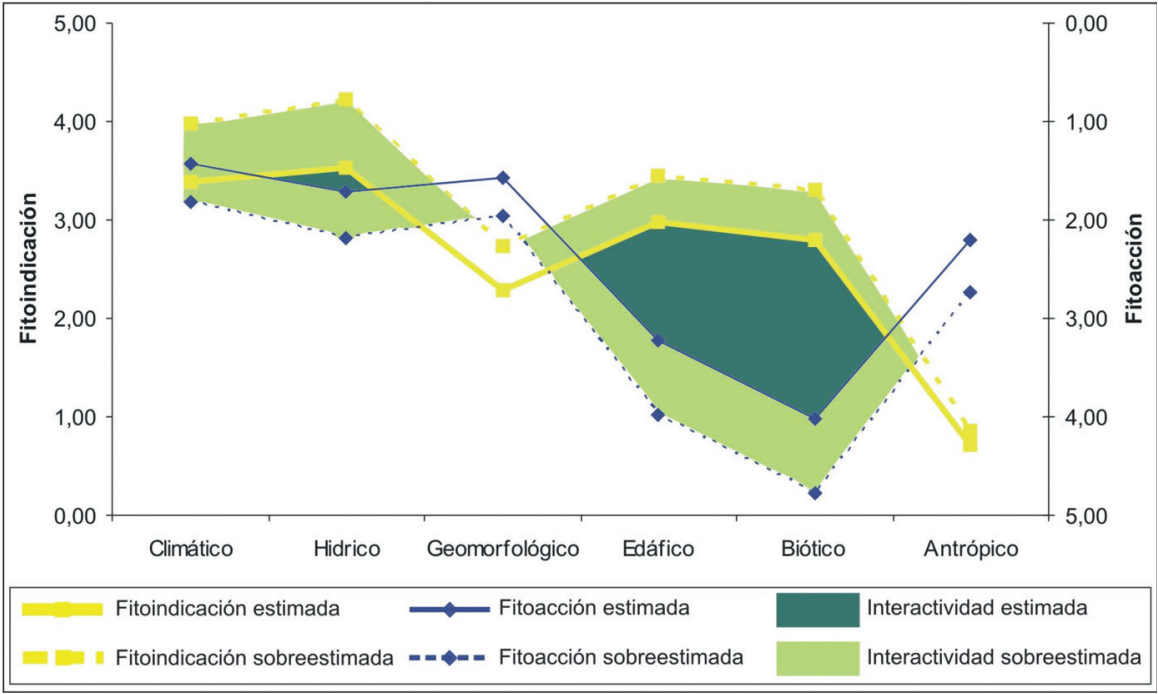

Fig. 4.- Continuación.

Fig. 4.- Continuation. 
nico. Permite conocer sobre qué factores reside la funcionalidad geoecológica en función de las relaciones que cada taxón (poblaciones) establece con cada uno de los elementos agrupados para la escala de análisis propuesta en el papel que todo ser vivo adquiere, tanto como sujeto pasivo (determinado por los factores), como activo (condicionante de tales factores) y que éstos difieren en una misma comunidad a lo largo de sus diferentes etapas sucesionales, tanto en el tipo de factores, como en su grado de incidencia o intensidad.

La versión última de esta metodología permite que sus resultados puedan ser asimilados y testados por otras herramientas y metodologías, como el caso expuesto de la utilización del test de significación estadística $t$ de Student y el análisis de la varianza (ANOVA) que no ha hecho sino reforzar la validez de los resultados obtenidos por el "Método EFG-Meaza" y corroborar la argumentación que explica la sucesión vegetal del caso estudiado

\section{Agradecimientos}

Agradecer a Itxaro Latasa por sus aportaciones cartográficas y a los revisores de la revista Munibe Ciencias Naturales por su contribución a la mejora del presente trabajo.

\section{Bibliografía}

- Braun-Blanquet, J. 1978. Fitosociología. Bases para el estudio de las comunidades vegetales. H. Blume editores. Madrid.

- Gómez, D.C., Meaza, G. 2010.Interactividad fitoindicación/fitoacción: aplicación en fresnedas de los ríos Cerjena (Burgos) y Guadaíra (Sevilla). Lurralde 33: 15-36.

- Gómez, D.C., Meaza, G., Cámara, R. 2010. Aplicación del Método Fi/Fa al bosque de ribera del Arroyo La Madre (Cuenca del Guadaíra. Sevilla). En Biogeografía: una ciencia para la conservación del medio. VI Congreso Español de Biogeografía. P. Giménez, J.A. Marco, E. Matarredona, A. Padilla y A. Sánchez (Eds.): 233-244. Universidad de Alicante, Departamento de Análisis Geográfico Regional y Geografía Física. Alicante.

- Gómez, D.C., Meaza, G. 2013. Funcionalidad geoecológica del tarajal del arroyo Aceitero (Alto Guadaira, Sevilla). Lurralde 36: 51-66.

- Gómez, D.C., Laguna, E., Meaza, G. 2014. Dinámica y caracterización geoecológica de comunidades vegetales a partir de valores indicadores de Ellenberg y del método Fitoindicación/Fitoacción. Ensayo de aplicación en los tarajales del arroyo aceitero (Cuenca del Guadaira, Sevilla). Geographicalia 65: 87-114.

- Gómez, D.C., Lozano, P.J., Meaza, G. 2015. Dynamics of riparian vegetation (Geo-Ecodynamics) in Thermo-Mediterranean landscapes. In: Fluvial Geomorphology and Riparian Vegetation: Environmental Importance, Functions and Effects of Climate Change. N. Duncan (Eds.): 1-52. Nova Science Publishers, Inc. Hauppauge. New York. 
- Gonzalez, F. 1982. Análisis ecosistémico de los recursos naturales. Fascículos sobre medio ambiente. No2. CIFCA. Madrid.

- Hammer, Ø. 2014. PAST 3.x - the Past of the future. Paquete estadístico, Oslo, University of Oslo, Natural History Museum. Disponible en: http://folk.uio.no/ohammer/past/index.html. (Consulta: 10.11 .2014$)$

- Hammer, Ø. Harper, D.A.T., Ryan, P.D. 2005. PAST-Paleontological statistics, ver. 1.37.

- Laguna, E. 1995.Fenología de la flora y vegetación de la serie del carrascal basófilo mesomediterráneo en la umbría del Fresnal de Buñol (Sierra de Malacara, Valencia). Universitat de València, Servei de Publicacions. Valencia. (Serie Tesis Doctorales en Microfichas, nº 055-21).

- Laguna, E. 1997.Vegetación y flora de la Umbía del Fresnal (Sierra de Malacara, Hoya de BuñolChiva). Buñol (Valencia). Instituto de Estudios Comarcales Hoya de Buñol-Chiva.

- Laguna, E. 2012. El método fitosociológico aplicado al seguimiento de la evolución de la flora en espacios protegidos: el caso de Limonium dufourii en la microreserva de flora 'Cap de Cullera' (Valencia). En: Las zonas de montaña: Gestión y Biodiversidad. VII Congreso Español de Biogeografía. R. Cunill, A. Pélachs, R. Pérez-Obiol y J.M. Soriano, (Eds): 262-268. Universitat Autònoma de Barcelona, Departament de Geografia. Barcelona.

- Laguna, E., Ferrer-Gallego, P.P. 2015. Propuestas de aplicación de los índices de diversidad para usos taxonómicos, fitosociológicos y listas rojas de flora amenazada. Flora Montiberica 60: 18-31.

- Laguna, E., Ferrer, P.P., Gómez, D.C., Lozano, P.J., Meaza, G. 2015a. Datos sobre la evolución de los "fresnales" o fresnedas de flor valencianas, obtenidos mediante el inventariado diacrónico. Geographicalia 67: 77-105.

- Laguna, E., Ferrer, P.P., Gómez, D.C., Lozano, P.J., Meaza, G. 2015b. Evolución de comunidades singulares o amenazadas: el caso de las fresnedas de flor valencianas. Edit. SEBICOP-ARANZADI. Libro de los resúmenes del VII Congreso de Biología de la conservación de plantas. VitoriaGasteiz.

- Lozano, P.J., Gómez, D.C., Meaza, G. 2012. Interactividad Bioindicación/Bioacción y funcionalidad geoecológica. Ensayo de aplicación en un encinar basófilo de Espinosa de Los Monteros (Burgos, España). En: Las zonas de Montaña: gestión y biodiversidad. VII Congreso Español de Biogeografía. R. Cunill, A. Pélachs, R. Pérez-Obiol y J.M. Soriano, (Eds): 219-225. Universitat Autònoma de Barcelona, Departament de Geografia. Barcelona.

- Meaza, G. 1993. Propuesta metodológica de valoración fitogeográfica de unidades de paisaje vegetal. En: Libro Homenaje al investigador Felix M. Ugarte Elorza. 369-391. Bilbao.

- Meaza, G. (Coord.) 2000. Metodología y Práctica de la Biogeografía. Ediciones del Serbal. Barcelona.

- Meaza, G., Cadiñanos, J.A. 2000. Valoración de la Vegetación. En: Metodología y Práctica de la Biogeografía. G. Meaza (Ed.): 201-272. Ediciones del Serbal. Barcelona.

- Meaza, G., Cuesta, M.J. 2010. Fitoindicación/fitoacción ambiental y territorial. Ensayo de aplicación en la Reserva de la Biosfera de Urdaibai (País Vasco). Bol. Asoc. Geógrafos Esp. 54: 99123. 
- Sokal, R.R., Rohlf, F.J. 1994. Biometry: The principles and practice of statistics in biological research. WH Freeman \& Co. London.

- Rivas-Martínez, S. 2011. Mapa de series, geoseries y geopermaseries [Memoria del mapa de vegetación potencial de España], parte II. Itinera Geobotanica 18(1): 5-424.

- Rivas-Martínez, S., Fernández-Gonzalez, F., Loidi, J., Lousa, M., Penas, A. 2001. Syntaxonomical checklist of vascular plant communities of Spain and Portugal Association Lebel. Itinera Geobotánica 14: 5-341.

- Rivas-Martínez, S. (Coord.) 2011. Mapa de series, geoseries y geopermaseries de vegetación de España [Memoria del Mapa de Vegetación Potencial de España, parte II]. Itinera Geobotánica 18(1): 1-425. 